\title{
CONSTRAINTS ON PARTICLE ACCELERATION FROM THE EXTRAGALACTIC $\gamma$-RAY BACKGROUND
}

\author{
K. MANNHEIM \\ Universitäts-Sternwarte \\ Geismarlandstraße 11, D-37083 Göttingen
}

\begin{abstract}
The propagation of $\gamma$-rays through metagalactic space is associated with pair creation and subsequent inverse-Compton scattering off low-energy background radiation. As a consequence, $\gamma$-rays of very high energy emitted by remote sources are reprocessed into the window from $10 \mathrm{MeV}$ to $30 \mathrm{GeV}$ conserving the injected energy. Any cosmologically distributed population of $\gamma$-ray sources therefore contributes to the diffuse $\gamma$-ray background in this energy band which is well-determined from recent observations with the Compton Gamma Ray Observatory (CGRO). Since the $\gamma$-rays trace accelerated particles, the observed flux of diffuse $\gamma$-rays also constrains the global efficiency for particle acceleration. Radio galaxies can account for the $\gamma$-ray background if their particle acceleration efficiency considerably exceeds $\sim 18 \%$ implying that particle acceleration is an essential part of the thermodynamics in these sources.
\end{abstract}

\section{Introduction}

The microphysics of particle acceleration in the solar system is a fascinating realm and provides useful paradigms for particle acceleration in other places of the Universe. From the observed flux of cosmic rays it is clear that much more powerful particle accelerators than main sequence stars and their winds must be at work in our Galaxy and in other galaxies. Supernova remnants (Ellison et al., 1997) and radio galaxies (Biermann, 1995) are among the most interesting putative sources of cosmic rays. The cosmic ray pressure is rather large and compares with that of the interstellar magnetic fields implying that cosmic rays are an important ingredient in the dynamics of the interstellar medium, and possibly also in the dynamics of the intergalactic medium (in superclusters). This imposes strong constraints on the energetics of possible cosmic ray sources from which one 
can infer the particle acceleration efficiency. For instance, supernova blast waves can supply the flux of cosmic rays up to the so-called knee in their spectrum at an energy of $10^{15} \mathrm{eV}$ only if their particle acceleration efficiency is as large as $\sim 13 \%$ (Drury, 1990) which basically invalidates simple testparticle approaches for the description of the acceleration mechanism. This has enforced the two-fluid theory for shock acceleration in which the momentum flux due to accelerated particles is self-consistently included into the dynamics of the shock wave (Achterberg et al., 1984). In this contribution it is argued that if radio galaxies are the most powerful particle accelerators in the Universe responsible for the diffuse isotropic gamma-ray background, and possibly also for the ultrahigh-energy cosmic rays up to at least $5 \times 10^{19} \mathrm{eV}$ (the so-called Greisen-Zatsepin-Kuzmin cutoff), they require a particle acceleration efficiency even larger than that of supernova remnants.

\section{Energy density of the diffuse isotropic $\gamma$-ray background}

The energy losses of relativistic particles inevitably lead to $\gamma$-radiation by which the acceleration sites can be traced. The cumulative flux from all unresolved cosmic accelerators appears as an diffuse isotropic flux in an inertial frame. A diffuse high-latitude $\gamma$-ray background has been measured with CGRO from $\mathrm{MeV}$ to $\sim 100 \mathrm{GeV}$ photon energies which very likely arises from unresolved extragalactic sources (Sreekumar et al., 1998). The resolved sources already contribute $\sim 15 \%$ of the diffuse isotropic flux and show the same average spectral shape as the diffuse background. The background spectrum above $30 \mathrm{MeV}$ as determined with EGRET on board CGRO is given by

$$
\frac{d N}{d E}=(7.32 \pm 0.34) \times 10^{-9}\left(\frac{E}{451 \mathrm{MeV}}\right)^{-2.1 \pm 0.03} \mathrm{~cm}^{-2} \mathrm{~s}^{-1} \mathrm{MeV}^{-1} \mathrm{sr}^{-1}
$$

The spectrum continues into the $\mathrm{MeV}$ range. Most of the background flux below $10 \mathrm{MeV}$ can be explained by the contribution of Supernovae Ia from the era of galaxy formation at $z_{\mathrm{f}}=2-5$ (The et al., 1993). The uncertainties in this thermal component of the diffuse $\gamma$-ray background are not very large, the known element abundances tightly constrain the total number of SNIa, and hence the total energy flux in $\gamma$-rays, in the Universe leaving little room for other sources contributing in the $\mathrm{MeV}$ range. The uncertainty in the cosmic star formation history due to possible dust enshrouding of the earliest galaxies should amount to less than a factor of three. Integrating the above non-thermal background spectrum between $10 \mathrm{MeV}$ and $30 \mathrm{GeV}$ (the flux at higher energies must be considered somewhat unreliable due to 
Constraints on particle acceleration from the extragalactic $\gamma$-ray background 3

the strongly decreasing aperture of EGRET) one obtains the energy density

$$
u_{\gamma}=(5.03 \pm 0.64) \times 10^{-6} \mathrm{eVcm}^{-3}
$$

or

$$
\Omega_{\gamma} h^{2}=(2.09 \pm 0.27) \times 10^{-10}
$$

where $h=H_{\circ} / 100 \mathrm{~km} \mathrm{~s}^{-1} \mathrm{Mpc}^{-1}$ parametrizes the Hubble constant.

\section{Metagalactic gamma-ray absorption and production}

Calorimetry of the particles accelerated throughout the Universe requires to know whether all $\gamma$-rays emitted by a source also reach the present-day observer. However, $\gamma$-rays of energy $E$ can interact with low-energy photons of energy $\epsilon$ from the diffuse isotropic background over cosmological distance scales $l$ producing electron-positron pairs $\gamma+\gamma \rightarrow e^{+}+e^{-}$, if their energy exceeds the threshold energy

$$
\epsilon_{\mathrm{th}}=\frac{2\left(m_{\mathrm{e}} c^{2}\right)^{2}}{(1-\mu)(1+z)^{2} E} \sim 1\left(\frac{1+z}{4}\right)^{-2}\left(\frac{E}{30 \mathrm{GeV}}\right)^{-1} \mathrm{eV} \quad(\mu=0)
$$

where $\mu$ denotes the cosine of the scattering angle (Gould \& Shréder, 1966; Coppi \& Aharonian, 1997). The $\gamma$-ray attenuation $e^{-\tau}$ due to pair production becomes important if the mean free path $\lambda$ becomes smaller than $l$, i.e. if the optical depth obeys $\tau=l / \lambda \geq 1$. For the computation of $\tau$ one first needs to know the pair production cross section

$$
\sigma_{\gamma \gamma}=\frac{3 \sigma_{\mathrm{T}}}{16}\left(1-\beta^{2}\right)\left[2 \beta\left(\beta^{2}-2\right)+\left(3-\beta^{4}\right) \ln \left(\frac{1+\beta}{1-\beta}\right)\right]
$$

where $\beta=\sqrt{1-1 / \gamma^{2}}$ with $\gamma^{2}=\epsilon / \epsilon_{\mathrm{th}}$, and where $\sigma_{\mathrm{T}}$ denotes the Thomson cross section (Jauch \& Rohrlich, 1976). Then one needs the geodesic radial displacement function $d l / d z=\frac{c}{H_{\circ}}[(1+z) \bar{E}(z)]^{-1}$ to compute the line integral from $z=0$ to some $z=z_{\mathrm{o}}$. For a cosmological model with $\Omega=1$ and $\Lambda=0$ the function $\bar{E}(z)$ simplifies to $(1+z)^{3 / 2}$. Hence one obtains the optical depth

$$
\begin{aligned}
\tau_{\gamma \gamma}\left(E, z_{\circ}\right) & =\int_{0}^{z_{\circ}} d z \frac{d l}{d z} \int_{-1}^{+1} d \mu \frac{1-\mu}{2} \int_{\epsilon_{\mathrm{th}}}^{\infty} d \epsilon n_{\mathrm{b}}(\epsilon)(1+z)^{3} \sigma_{\gamma \gamma}(E, \epsilon, \mu, z) \\
= & \frac{c}{H_{\circ}} \int_{0}^{z_{\circ}} d z(1+z)^{1 / 2} \int_{0}^{2} d x \frac{x}{2} \int_{\epsilon_{\mathrm{th}}}^{\infty} d \epsilon n_{\mathrm{b}}(\epsilon) \sigma_{\gamma \gamma}(E, \epsilon, x-1, z)
\end{aligned}
$$

adopting a non-evolving present-day background density $n_{\mathrm{b}}$, i.e. $n_{\mathrm{b}}^{\prime}\left(z, \epsilon^{\prime}\right) d \epsilon^{\prime}=$ $(1+z)^{3} n_{\mathrm{b}}(\epsilon) d \epsilon$ where the dash indicates comoving-frame quantities. The 


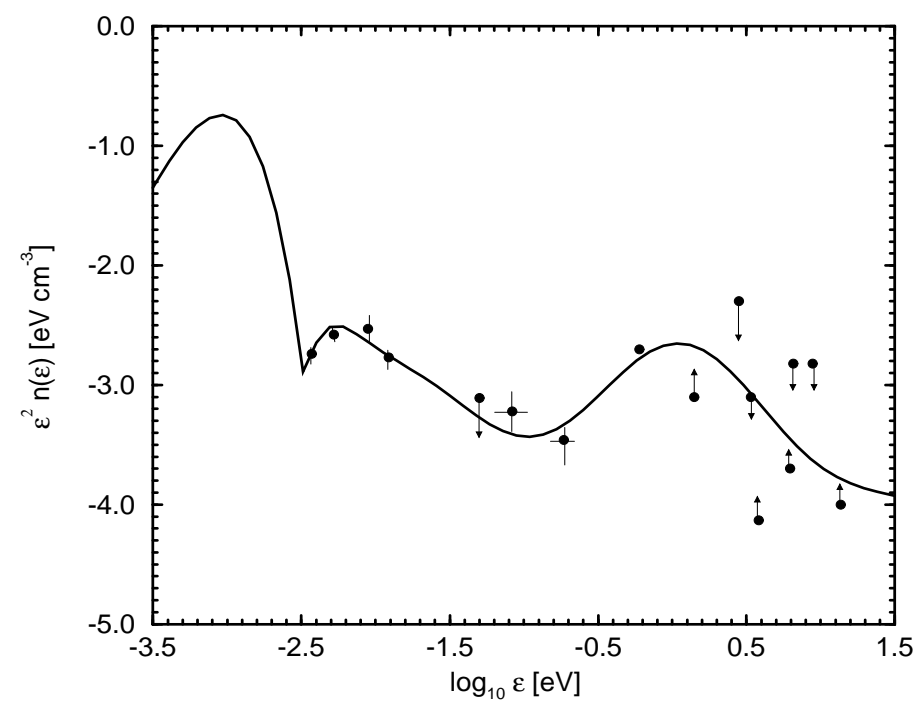

Figure 1. The diffuse isotropic microwave-to-ultraviolet background: the solid curve shows a 10th order polynomial interpolation of observational data ((Fixsen et al., 1998; Mannheim, 1998; Franceschini et al., 1998), and references in (Madau \& Pinney, 1996)).

simplifying assumption that the photon density transforms geometrically corresponds to a situation in which an initial short burst of star formation at $z_{\mathrm{f}}>z_{\circ}$ produced most of the diffuse infrared-to-ultraviolet background radiation. Fig.1 shows the spectrum of the low-energy diffuse background used to solve Eq.(6) numerically.

Figure 2 shows the resulting $\tau(E, z)=1$ (omitting the subscript hereafter) curve for the microwave-to-ultraviolet diffuse background spectrum shown in Fig.1. It is obvious that $\gamma$-rays above $\sim 10-50 \mathrm{GeV}$ can not reach us from beyond redshifts of $z=z_{\mathrm{f}}=2-4$. Higher energy $\gamma$-rays can reach us only from sources at lower redshifts (e.g. $\gamma$-rays with energies up to $10 \mathrm{TeV}$ have been observed from Mrk 501 at $z=0.033$ in accord with Fig.2 (Mannheim, 1998)).

Corollary I: If the DIGB originates from unresolved sources distributed in redshift similar to galaxies, its spectrum must steepen above $\sim 30 \mathrm{GeV}$ due to $\gamma$-ray pair attenuation.

Here is has been tacitly assumed that the $\gamma$-rays which have turned into electron-positron pairs do not show up again. This is, in fact, not quite true, since the pairs are subject to inverse-Compton scattering off the 
Constraints on particle acceleration from the extragalactic $\gamma$-ray background 5

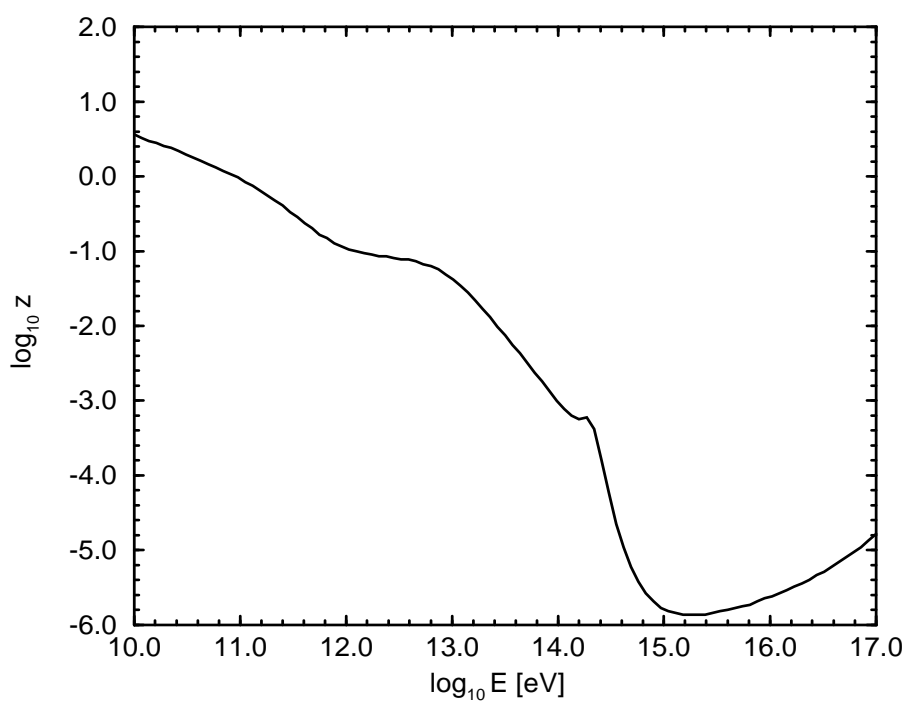

Figure 2. The $\gamma$-ray horizon $\tau(E, z)=1$ for the low-energy background spectrum shown in Fig.1. Cosmological parameters are $h=0.6, \Omega=1$, and $\Omega_{\Lambda}=0$. For a general discussion of pair attenuation, see reference (Biller, 1996).

microwave background thereby replenishing $\gamma$-rays. The $2.7 \mathrm{~K}$ background is more important as a target than the shorter wavelength background, since there is no threshold condition for Thomson scattering contrary to pair production and since $2.7 \mathrm{~K}$ photons greatly outnumber the latter. The inverse-Compton scattered microwave photons turn into $\gamma$-rays of energy

$$
E_{\mathrm{ic}} \sim 10\left(\frac{1+z}{4}\right)\left(\frac{E}{30 \mathrm{GeV}}\right)^{2} \mathrm{MeV}
$$

conserving the energy of the absorbed $\gamma$-ray which corresponds to a constant $E^{2} d N / d E$, i.e. the expected slope of the differential spectrum is about -2 (-2.1 observed). A negligible amount of energy is lost to lower frequency synchrotron emission, if magnetic fields are present in the interagalactic medium.

Corollary II: Energy conservation in the reprocessing of $\gamma$-rays from higher to lower energies by pair production and subsequent inverse-Compton scattering produces an approximate $d N / d E \propto E^{-2}$ power law DIGB between $\sim 10 \mathrm{MeV}$ and $\sim 30 \mathrm{GeV}$. 


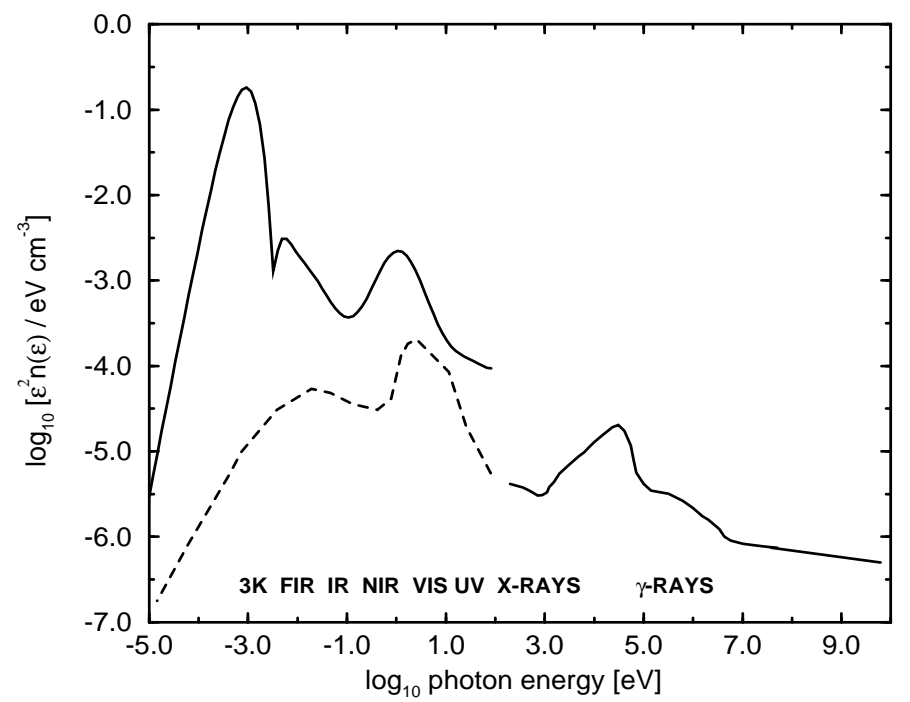

Figure 3. Extragalactic background radiation. The solid curve interpolates observational data. Whereas AGN dominate in the X-ray regime, their longer wavelength emission (dotted line from (Sanders et al., 1989)) is surpassed by the stellar and dust emission from early galaxies.

\section{Particle acceleration efficiency}

The energy density of the accelerated particles in the sources $\left(u_{\text {acc }}\right)$ must obey $u_{\text {acc }} \geq u_{\gamma}$ for consistency. Defining the radiative efficiency

$$
\xi_{\mathrm{rad}}=\frac{u_{\gamma}}{u_{\mathrm{acc}}}=\operatorname{Min}\left[1, \frac{t_{\mathrm{a}}}{t_{\mathrm{c}}}\right]
$$

where $t_{\mathrm{a}}$ and $t_{\mathrm{c}}$ denote the adiabatic and energy loss time scales, respectively, the energy requirement for the sources is minimized for $\xi_{\text {rad }}=1$. In extragalactic radio sources, $\xi_{\text {rad }} \sim 1$ corresponds to very large electron $\left(\gamma_{\mathrm{e}} \sim 10^{5}\right)$ or proton Lorentz factors $\left(\gamma_{\mathrm{p}} \sim 10^{10}\right)$ consistent with the nonthermal $\gamma$-ray spectra extending above $10 \mathrm{GeV}$.

The resolved extragalactic $\gamma$-ray sources belong to the so-called blazar subclass of radio-loud active galactic nuclei (AGN) in which one observes jets emerging from accreting supermassive black holes preferentially at small angles to their relativistic velocity vector. If the entire class of radioloud AGN is responsible for the DIGB, the particle acceleration efficiency is constrained by

$$
\xi_{\mathrm{acc}}=\frac{u_{\mathrm{acc}}}{u_{\mathrm{j}}}=\frac{u_{\gamma}}{\xi_{\mathrm{rad}} u_{\mathrm{j}}}
$$


Constraints on particle acceleration from the extragalactic $\gamma$-ray background 7

where $u_{\mathrm{j}}$ denotes the total (kinetic + magnetic + randomized relativistic particle) energy density in extragalactic jets. Since $u_{\gamma}$ is known (Eq.(1)), a determination of $u_{\mathrm{j}}$ is necessary to further constrain $\xi_{\text {acc }}$. This energy density can be inferred from the relative strengths of the various bumps in the overall diffuse background spectrum (Fig.3) in the following way.

\section{Origins of the diffuse isotropic background radiation}

The microwave bump is the well-known signature of the big bang at the time of decoupling with its energy density given by the Stefan-Boltzmann law $u_{3 \mathrm{~K}}=\sigma T^{4}$. The bump in the far-infrared is due to star formation in early galaxies (at $z=z_{\mathrm{f}}$ ), since part of the stellar light, which is visible as the bump at visible wavelengths, is reprocessed by dust obscuring the star-forming regions. The energy density of the two bumps can be inferred from the present-day heavy element abundances. Heavy elements have a mass fraction $Z=0.03$ and were produced in early bursts of star formation by nucleosynthesis with radiative efficiency $\epsilon=0.007$ yielding

$$
u_{\mathrm{ns}} \sim \frac{\rho_{*} Z \epsilon c^{2}}{1+z_{\mathrm{f}}} \sim 6 \times 10^{-3}\left(\frac{\Omega_{*} h^{2}}{0.01}\right)\left(\frac{1+z_{\mathrm{f}}}{4}\right)^{-1} \mathrm{eV} \mathrm{cm}^{-3}
$$

About half of the energy is contained in either bump. It has been shown recently by a number of groups that probably all galaxies (except dwarfs) contain supermassive black holes in their centers which are actively accreting over a fraction of $t_{\mathrm{agn}} / t_{*} \sim 10^{-2}$ of their lifetime implying that the

electromagnetic radiation released by the accreting black holes amounts to

$$
u_{\mathrm{accr}} \sim \frac{\epsilon_{\mathrm{accr}} M_{\mathrm{bh}}}{Z \epsilon M_{*}} \frac{t_{\mathrm{agn}}}{t_{*}} u_{\mathrm{ns}} \sim 1.4 \times 10^{-4} \mathrm{eV} \mathrm{cm}^{-3}
$$

adopting the accretion efficiency $\epsilon_{\text {accr }}=0.1$ and the black hole mass fraction $M_{\mathrm{bh}} / M_{*}=0.005$ (Rees \& Silk, 1998). Most of the accretion power emerges in the ultraviolet where the diffuse background is unobservable owing to photoelectric absorption by the neutral component of the interstellar medium. However, a fraction of $u_{\mathrm{x}} / u_{\mathrm{bh}} \sim 20 \%$ (from the average quasar spectral energy distribution (Sanders et al., 1989)) shows up in hard Xrays due to coronal emission from the accretion disk to produce the diffuse isotropic X-ray background bump with $u_{\mathrm{x}} \sim 2.8 \times 10^{-5} \mathrm{eV} \mathrm{cm}^{-3}$ (Gruber, 1992).

Jets with non-thermal ( $\gamma$-ray) emission show up only in the radio-loud fraction $\xi_{\mathrm{rl}} \sim 20 \%$ of all AGN and their kinetic power roughly equals the accretion power (Rawlings \& Saunders, 1991). Hence one obtains for the energy density in extragalactic jets

$$
u_{\mathrm{j}}=\left(\frac{\xi_{\mathrm{rl}}}{0.2}\right) u_{\mathrm{accr}} \sim\left(\frac{\xi_{\mathrm{rl}}}{0.2}\right) 2.8 \times 10^{-5} \mathrm{eV} \mathrm{cm}^{-3}
$$


Substituting this value into Eq.(9) one obtains a limit for the acceleration efficiency

$$
\xi_{\text {acc }} \geq 0.18\left(\frac{\xi_{\text {rad }}}{1.0}\right)^{-1}\left(\frac{\xi_{\text {rl }}}{0.2}\right)^{-1}
$$

which has to be compared with the $13 \%$ efficiency required for supernova remnants. Since a radiative efficiency much lower than unity must be considered realistically (e.g., to account for the pdV work of the jets against a surrounding intracluster medium), the acceleration efficiency may have to be as large as $30 \%$ or more. Possible escapes to this conclusion are (i) the radio-loud fraction was much larger at high redshifts than at low redshifts, (ii) there is another contributor to the gamma-ray background, such as the decay of topological defects (Sigl, 1996), or (iii) the flux of the DIGB is actually lower due to the foreground from a galactic halo (Dixson et al., 1998).

\section{A remark on cosmic rays from radio galaxies}

An extragalactic cosmic ray component seems to be evident from the observed change in slope of the local spectrum above $3 \times 10^{18} \mathrm{eV}$. The slope of this extragalactic component is much steeper than $E^{-2}$ consistent with the steepening due to energy losses for a cosmologically distributed and evolving source population, such as extragalactic radio sources (Rachen et al., 1993). The total energy density in the loss-steepened extragalactic cosmic ray component and the DIGB are of the same order of magnitude, as required if the $\gamma$-rays trace neutral pions and the cosmic rays trace neutrons produced in photo-meson production events.

\section{Summary}

The particle acceleration efficiency of the jets in radio galaxies must be larger than $\sim 18 \%$ if the $\gamma$-rays from these jets are to explain the DIGB.

\section{References}

Achterberg, A., Blandford, R., Periwal, V., 1984, A\&A, 132, 97

Biermann, P.L., 1995, Sp.Sc.Rv., 74, 385

Biller, S.D., 1996, A.Ph., 3, 385

Coppi, P.S., Aharonian, F., 1997, ApJ, 487, L9

Dixson, D.D., et al., 1998, New Astro., in press

Drury, L.O'C., 1990, 21st Int.Cos.Ray Conf., 6-19 Jan 1990, Adelaide, Au., p.85

Fixsen, D.J., et al., 1997, ApJ, 490, 482

Franceschini, A., et al., 1998, astroph/9707080

Gould, R.J., Shréder, G., 1966, Phys.Rev.Lett., 16, 252

Gruber, D.E., 1992, in The X-ray Background, X. Barcons, A.C. Fabian, Cambridge, 44

Jauch, J.M., Rohrlich, F., 1976, The Theory of Photons and Electrons, Springer, Berlin 
Constraints on particle acceleration from the extragalactic $\gamma$-ray background 9

Madau, P., Phinney, E.S., 1996, ApJ, 456, 124

Mannheim, K., 1998, Science, 279, 684

Ellison, D.C., Drury, L.O'C., Meyer, J.-P., 1997, ApJ, 487, 197

Rachen, J.P., Stanev, T., Biermann, P.L., 1993, A\&A A, 273, 377

Rawlings, S., Saunders, 1991, Nature, 349, 138

Rees, M.J., Silk, J., 1998, $A \& A$, 331, L1

Sanders, D.B., et al., 1989, ApJ, 347, 29

Sigl, G., 1996, S.S.Rv., 75, 375

Sreekumar, P., Stecker, F.W., Kappadath, S.C., 1998, astroph/9709258

The, L.-S., Leising, M.D., Clayton, D.D., 1993, ApJ, 403, 32 